\title{
Non-thermal radio supernova remnants of exiled Wolf-Rayet stars
}

\author{
D. M.-A. Meyer, ${ }^{a, *}$ M. Pohl, ${ }^{a, b}$ M. Petrov ${ }^{c}$ and L. Oskinova ${ }^{b, d}$ \\ ${ }^{a}$ Institut für Physik und Astronomie, Universität Potsdam, Karl-Liebknecht-Strasse 24/25, 14476 \\ Potsdam, Germany \\ ${ }^{b}$ DESY Platanenallee 6, D-15738 Zeuthen, Germany \\ ${ }^{c}$ Max Planck Computing and Data Facility (MPCDF), Gießenbachstrasse 2, D-85748 Garching, \\ Germany \\ ${ }^{d}$ Department of Astronomy, Kazan Federal University, Kremlevskaya Str 18, Kazan, Russia \\ E-mail: dmameyer.astro@gmail.com
}

When a massive star is swung away from the stellar cluster in which it forms, a so-called runaway stellar object is produced, and its surroundings is shaped as a bow shock which can be observed. Such arc-like circumstellar nebula is the site of the star's death via a supernova explosion, and, the interaction of the supernova shock wave with its surroundings results in an asymmetric supernova remnant. We investigate the synchrotron appearance of those supernova remnants, by means of $2 \mathrm{D}$ magneto-hydrodynamical simulations and radiative transfer calculations of the remnant left behind a $35 \mathrm{M}_{\odot}$ fast-moving star. The progenitor star evolves up to the Wolf-Rayet phase and the considered space motion through the magnetised ISM is of 20 and $40 \mathrm{~km} \mathrm{~s}^{-1}$, respectively. The magnetic field of the ISM has a stabilising effect regarding to the wind/ISM interface developing in the tail of the pre-supernova stellar wind bubble. Radiative transfer calculations for synchrotron radiation of our simulation models indicate that non-thermal radio emission has characteristic features accounting for the morphology of our exiled remnants of WolfRayet progenitor stars. The synthetic prediction are qualitatively in accordance with the non-thermal radio appearance of several remnants from massive progenitors such as CTB109, Kes 17 and G296.5+10.0.

$3^{\text {rth }}$ International Cosmic Ray Conference (ICRC 2021)

July 12th - 23rd, 2021

Online - Berlin, Germany

${ }^{*}$ Presenter 


\section{Introduction}

Wolf-Rayet stars are a possible final evolutionary phase of some $\geq 20 \mathrm{M}_{\odot}$ high-mass stars. A fraction of Wolf-Rayet stars fast-move through their local ambient medium after having been ejected from the stellar cluster they form in, and, sometimes, they can even travel to the high-galactic latitudes, low-density regions of the ISM [1-4]. At the end of their live, they experience a so-called core-collapse supernova explosion that happens inside of the circumstellar medium pre-shaped by the strong winds of the progenitor star [5-7]. Expelling strong, dense wind, runaway Wolf-Rayet-evolving stellar objects are first choice progenitor candidates for the production of asymmetric core-collapse supernova remnants [8].

Indeed, amongst the several mechanisms at work in the shaping of core-collapse supernova remnants, the gas distribution generated by the stellar wind of the progenitor with the ambient medium is of prime importance. Its level of complexity is proportional to the initial progenitor mass at the zero-age main-sequence phase, i.e. higher-mass stars evolve through a larger number of evolutionary phases, each of them releasing shells of enriched gas, such as the red supergiant and Wolf-Rayet materials, into their stellar surroundings [9]. Famous example of such core-collapse supernova remnants are the Cygnus Loop nebula [10], or RWC 86 [11], which both exhibit features that are in accordance with the characteristics of an off-centered supernova explosion inside of the stellar wind bubble of an evolved progenitor star animated by a fast, supersonic bulk motion.

In this study, we investigate the morphologies of supernova remnants from Wolf-Rayet stars. By means of radiative transfer calculations, we predict their non-thermal radio synchrotron emission maps. We detail the methods used to perform the simulations of synthetic emission of supernova remnants of a runaway high-mass $35 \mathrm{M}_{\odot}$ in Section 2. Our results for the remnant structure and their emission are in Section 3. We present our conclusions in Section 4.

\section{Methods}

We first simulate the interaction between the stellar wind of the moving progenitor star, then the supernova happening in it, and, finally, we estimate the emission properties of the supernova remnants for non-thermal synchrotron emission. The magnetohydrodynamical simulations are performed with the PLUTO code [12-14] in a 2D fashion, using an axisymmetric cylindrical coordinate system. We inject the stellar wind in a small region centered onto its origin, and, the stellar wind properties are time-dependently interpolated from pre-calculated evolutionary tracks [15]. The supernova remnant phase is calculated in releasing in the circumstellar medium at the pre-supernova phase a core-collapse supernova blastwave $[16,17]$. Several passive scalar tracers permit to separate the different material involved in our problem, i.e. the unperturbed ambient medium, stellar wind and supernova ejecta. Then, for representative selected times of the supernova remnant's evolution, we reconstruct 3D emissivity cubes using the recipe of [18] and perform line-of-sight integration with the RADMC-3D code [19] to obtain normalised non-thermal synchrotron emission maps. 


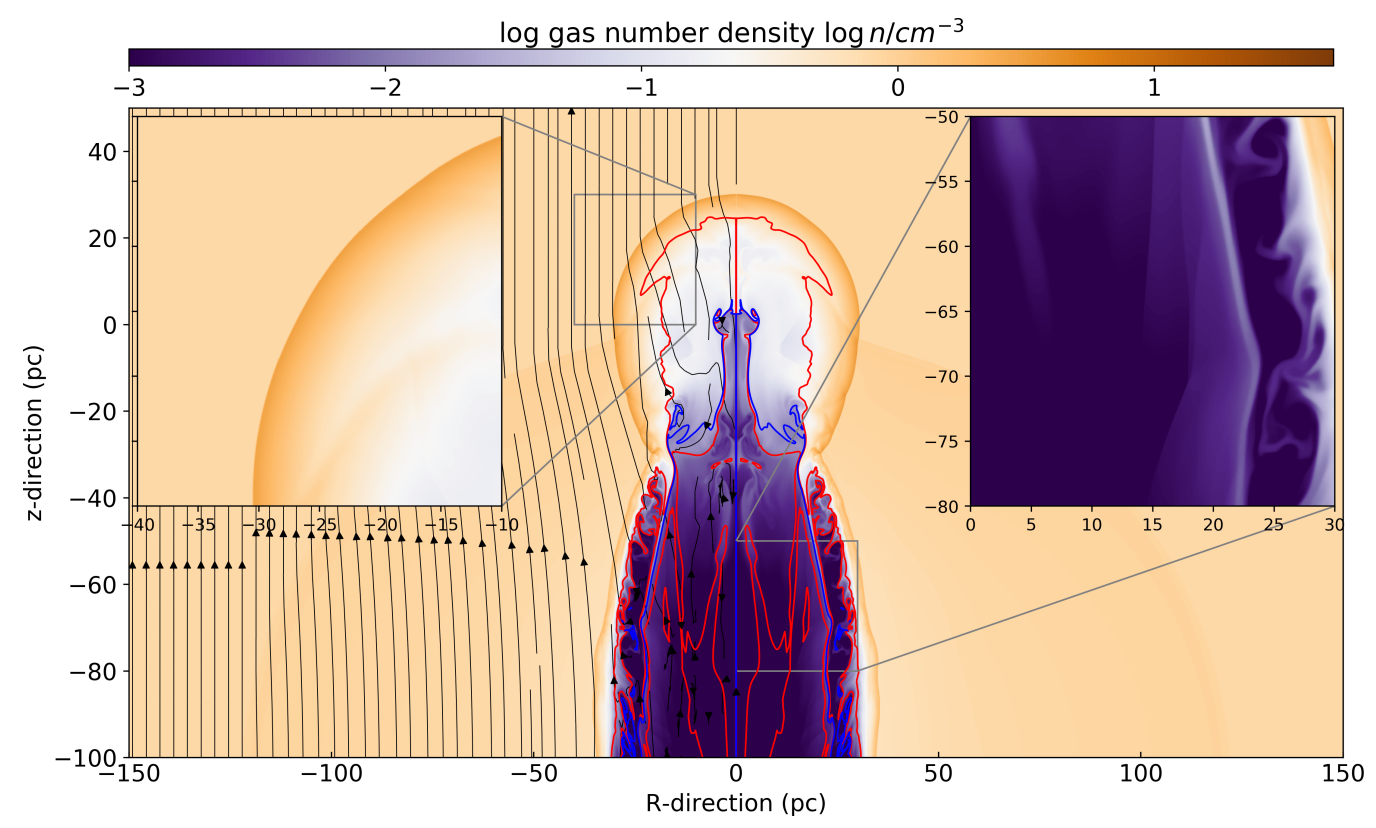

Figure 1: Number density field in our simulation of a supernova remnant of a runaway $35-\mathrm{M}_{\odot}$ star moving with $v_{\star}=20 \mathrm{~km} \mathrm{~s}^{-1}$ (see text for details).

\section{Results}

Fig. 1 shows the number density field of in our magneto-hydrodynamical model of a supernova remnant of a $35 \mathrm{M}_{\odot}$ progenitor runaway star moving with $v_{\star}=20 \mathrm{~km} \mathrm{~s}^{-1}$. The ambient medium in which the star moves is of number density $n_{\mathrm{ISM}}=0.78 \mathrm{~cm}^{-3}$ and its magnetization, parallel to the symmetry axis of the simulation and to the direction of stellar motion, along the $z$-axis, is of strength $7 \mu G$. The two inset boxes zoom on two particular regions, the filamentary ejecta-wind-ISM interactions (left inset) and the unstable walls of the wind cavity generated by the motion of the progenitor star (right inset). The red contours mark temperature contours for $T=10^{6}$ and $10^{7} \mathrm{~K}$, and, the blue lines highlight the location of the remnant with a $10 \%$ contribution of ejecta in number density. The stellar motion produced an elongated $\sim 70 \mathrm{pc}$ channel which pierced the wind bubble formed during the main-sequence phase of the star. The supernova shock wave has interacted with the dense bow shock of the progenitor ahead of the direction of stellar motion, while it expands freely in the opposite direction.

Fig. 2 is as Fig. 1 for our simulation model with $v_{\star}=40 \mathrm{~km} \mathrm{~s}^{-1}$. Since the progenitor star moves faster, its circumstellar medium adopts a cone-like shape rather than a large scale bow shock. The elongation of the cavity of stellar wind is more pronounced, and no trace of the pre-main-sequence stellar wind bubble remains within the $\sim 100$ pc-long trail of the stellar surroundings at the pre-supernova time. At the plotted time instance, the supernova shock wave has interacted against the walls of the cavity, creating long, dense filaments. It interacts with the former bow shock, expanding into the unperturbed ambient medium while recovering sphericity, and, it is quickly channelled into the unshocked wind. 


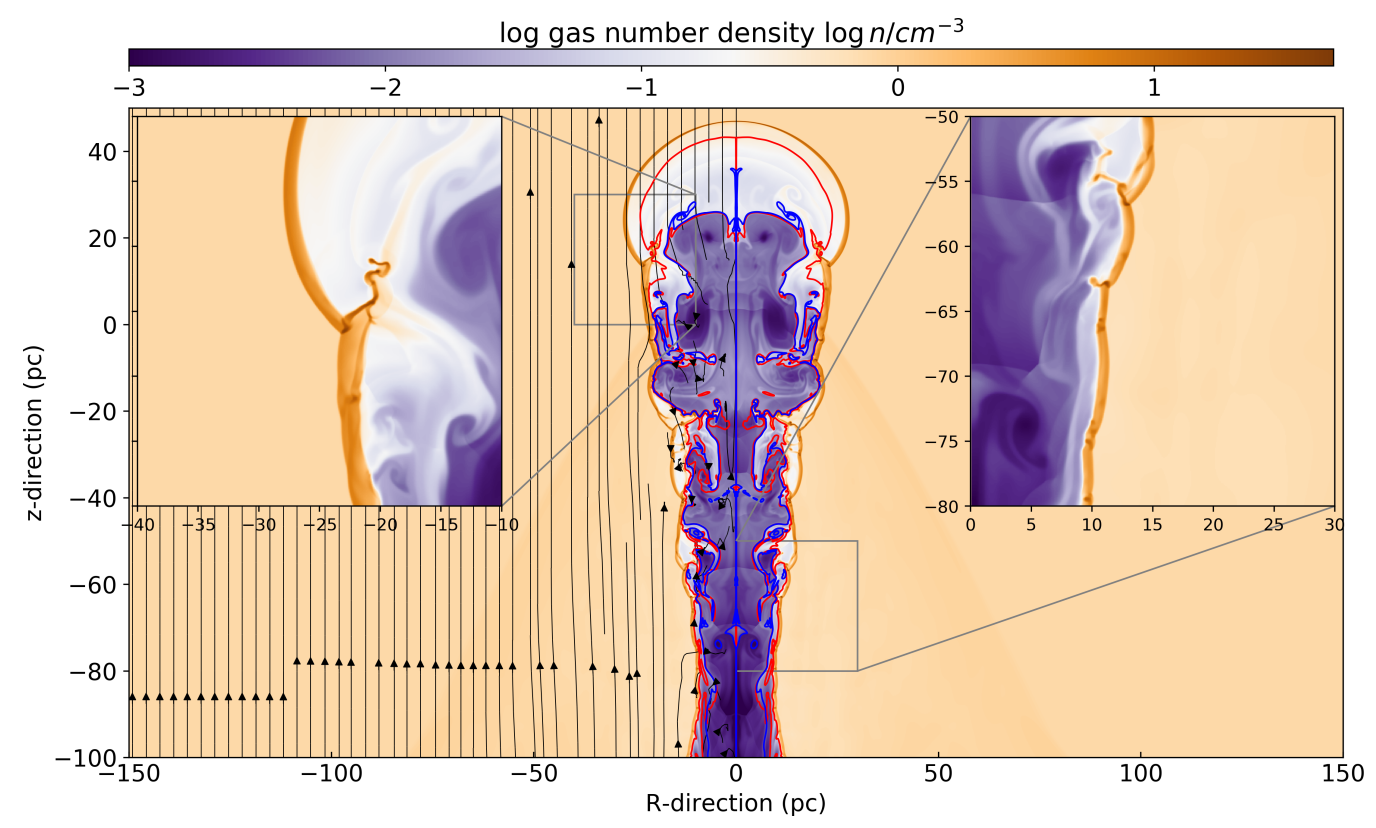

Figure 2: As Fig. 1 for our progenitor moving with $v_{\star}=40 \mathrm{~km} \mathrm{~s}^{-1}$.

In Fig. 3 we plot emission maps for non-thermal radio synchrotron emission of the remnant model with velocity $v_{\star}=20 \mathrm{~km} \mathrm{~s}^{-1}$, shown at several evolutionary times, and for different viewing angles, $\theta_{\mathrm{obs}}$. Once distorted by the interaction with its circumstellar medium, the supernova shock wave is well traced by the non-thermal radio radiation. When the expanding shock wave arrives at the unperturbed ISM, it becomes bright. The radio arcs are larger than at time $20 \mathrm{kyr}$ and the brightest region on the sides adopt a bilateral morphology. For an inclination angle $\theta_{\mathrm{obs}}=45^{\circ}$ the remnants look rounder. One and the same remnant can appear with bilateral or arced structures depending on the viewing angle. For $\theta_{\mathrm{obs}}=90^{\circ}$ the observer's line-of-sight is aligned with the direction of stellar motion, and the projected remnant appears as a ring-like structure in the sky, because of the $2 \mathrm{D}$ nature of the magneto-hydrodynamical models.

In Fig. 4 we show the emission maps corresponding to the simulation with progenitor speed $v_{\star}=40 \mathrm{~km} \mathrm{~s}^{-1}$. At time $6 \mathrm{kyr}$ after the explosion, the shock wave has already been greatly distorted by the Wolf-Rayet circumstellar material and has lost sphericity to become an ovoid-like structure. Later in time, the shock wave adopts a hour-glass-like shape that appears spherical in the radio map for $\theta_{\mathrm{obs}}=45^{\circ}$ and to a lesser degree at $\theta_{\mathrm{obs}}=0^{\circ}$. After $40 \mathrm{kyr}$, the remnant has an bulb-like morphology which arises from both the shock wave expansion into the ISM and the channeling of the shock wave into the low-density cavity of unshocked stellar wind in the tail. The radio intensity peak shifts to the location where the shock wave intercepts the trail of stellar wind (Fig. 4d,e). We would observe a series of concentric rings for $\theta_{\text {obs }}=90^{\circ}$, each of them corresponding to a ring in the trail of shocked stellar wind interacting with the channelled supernova shock wave. 


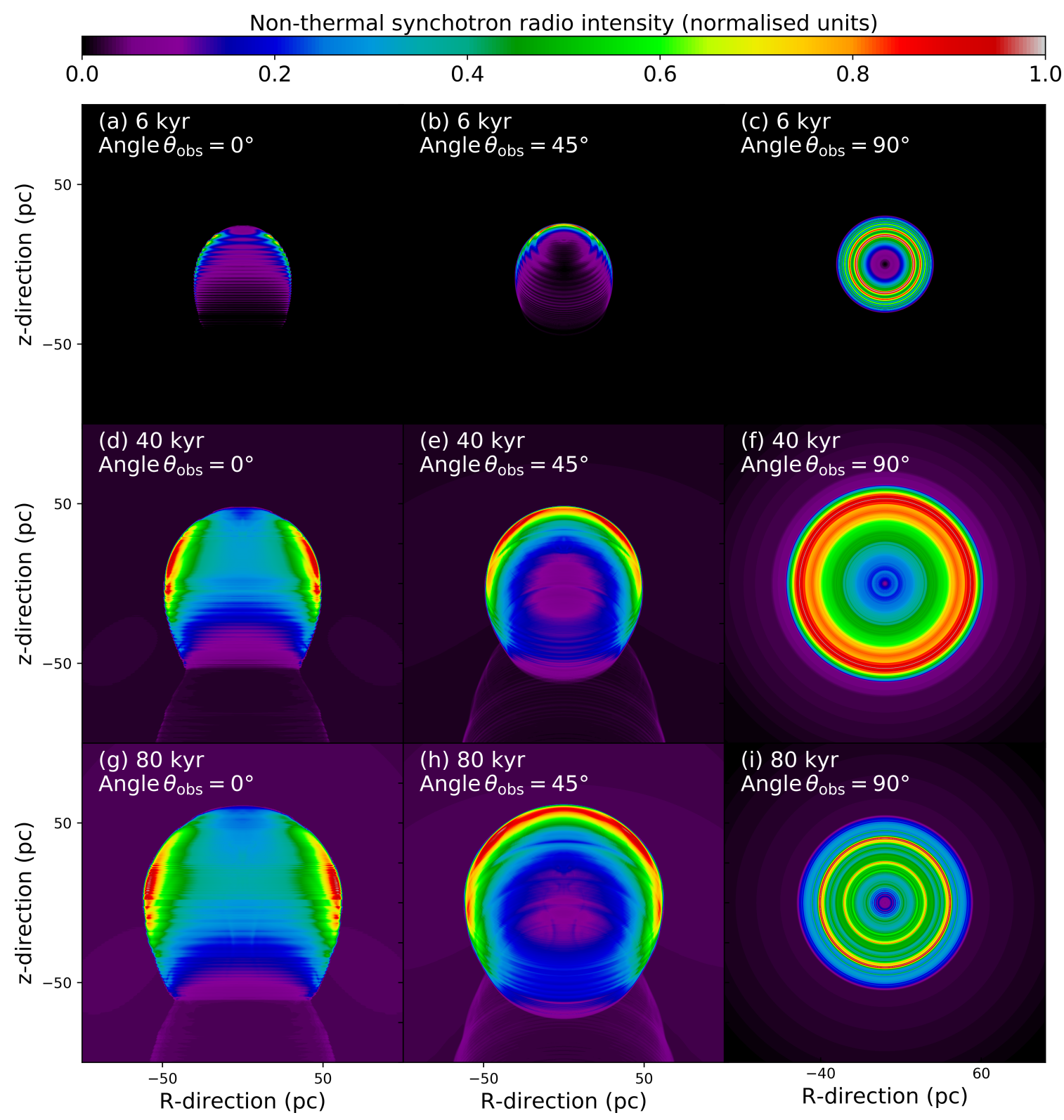

Figure 3: Emission maps of radio synchrotron intensity plotted in normalised units for our simulation of supernova remnants moving with velocity $v_{\star}=20 \mathrm{~km} \mathrm{~s}^{-1}$ at times $6 \mathrm{kyr}$ (top panels), $40 \mathrm{kyr}$ (middle panels), and $80 \mathrm{kyr}$ (bottom panels) after the explosion of the supernova, respectively. The viewing angle between is $\theta_{\mathrm{obs}}=0^{\circ}$ (left panels), $\theta_{\mathrm{obs}}=45^{\circ}$ (middle panels), and $\theta_{\mathrm{obs}}=90^{\circ}$ (right panels). 


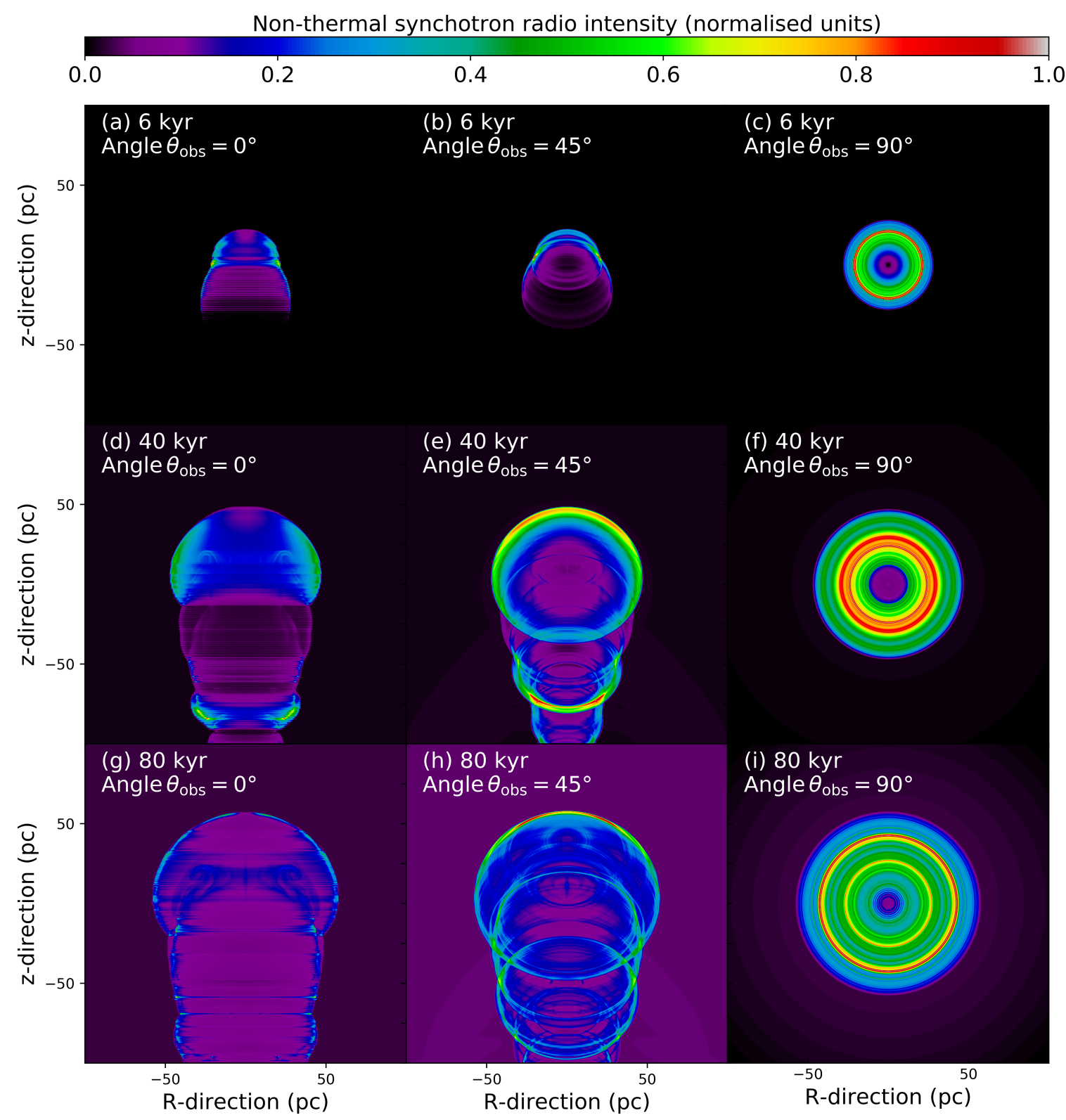

Figure 4: As for Fig. 1 for a stellar motion of $v_{\star}=40 \mathrm{~km} \mathrm{~s}^{-1}$.

Our models are qualitatively consistent with the radio appearance of several remnants of high-mass progenitors [8]. Particularly our model with $v_{\star}=20 \mathrm{~km} \mathrm{~s}^{-1}$ fits the bilateral supernova remnant G296.5 +10.0 [20] has been constrained to by expanding in magnetised ISM and to host a neutron star. The shell-type remnants CTB109 and Kes 17, of $30 \mathrm{M}_{\odot}$ and 20-40 $\mathrm{M}_{\odot}$ progenitors, respectively, are consistent with our simulation with $v_{\star}=40 \mathrm{~km} \mathrm{~s}^{-1}$ from which we produced shelled supernova remnants [21? ]. 


\section{Conclusion}

The explosion of a core-collapse explosion into the circumstellar medium of a runaway massive Wolf-Rayet star generates complex remnant nebula. It is made of a succession of magneto-hydrodynamical shocks, contact discontinuities between the different stellar winds of the defunct progenitor and supernova ejecta, respectively. The mixing of materials therein is efficient when the supernova blastwave is reverberated towards the center of the explosion after having interacted with the circumstellar medium of the progenitor. Such region are believed to the site location of non-thermal particles acceleration. Combining magneto-hydrodynamical simulations and radiative transfer calculations, we produce synthetic non-thermal radio-intensity maps revealing series of large-scale arcs and filaments in projection, which we interpret as being the typical characteristic of supernova remnants from runaway Wolf-Rayet stars. These radio emission maps are qualitatively similar to the morphology of many observed core-collapse supernova remnants like CTB 109, Kes 17 and G296.5 +10.0 which have probably been produced by $25-40 M_{\odot}$ stellar progenitors that might have experienced a Wolf-Rayet evolutionary phase [8].

\section{Acknowledgement}

The authors acknowledge the North-German Supercomputing Alliance (HLRN) for providing HPC resources that have contributed to the research results reported in this paper. MP acknowledges the Max Planck Computing and Data Facility (MPCDF) for providing data storage resources and HPC resources which contributed to test and optimize the PLUTO code. This research made use of the PLUTO code developed at the University of Torino by A. Mignone and collaborators, the MATPLOTLIB plotting library for the PYTHON programming language

\section{References}

[1] Munoz M., Moffat A. F. J., Hill G. M., Shenar T., Richardson N. D., Pablo H, St-Louis N. Ramiaramanantsoa T., 2017, MNRAS, 467, 3

[2] Toalá J. A., Oskinova L. M., Hamann W.-R., Ignace R. et al., 2018, MNRAS, 869, 1

[3] Moffat A. F. J., Marchenko S. V., Seggewiss W., van der Hucht et al., 1998, AAp, 331, 949-958

[4] Meyer, D. M. -A., Oskinova L. M., Pohl M., Petrov M., 2020, MNRAS, 496, 3

[5] Franco J., Tenorio-Tagle G., Bodenheimer P., Rozyczka M., 1991, PASP, 103, 803-810

[6] Rozyczka M., Tenorio-Tagle G., Franco J., Bodenheimer P., 1993, MNRAS, 261, 674680

[7] Dwarkadas V. V., 2007, ApJ, 667, 226-247 
[8] Katsuda S., Takiwaki T., Tominaga N., Moriya T. J., Nakamura K., 2018, ApJ, 863, 127

[9] Meyer D. M. -A., Pohl M., Petrov M., Oskinova L. M., 2021, MNRAS 502, 4

[10] Fang J., Yu H., Zhang L., 2017, MNRAS, 464

[11] Broersen S., Chiotellis A., Vink J., Bamba A., 2014, MNRAS, 441, 2

[12] Mignone A., Bodo G., Massaglia S., Matsakos T., Tesileanu O., Zanni, C. and Ferrari, A., 2007, ApJ, 170

[13] Mignone A., Zanni C., Tzeferacos P., van Straalen B., Colella P., Bodo G., 2012, ApJs\&A, 198

[14] Vaidya B., Mignone A., Bodo G., Rossi P., Massaglia S., 2018, ApJ, 865, 144

[15] Meyer D. M.-A., van Marle A.-J., Kuiper R., Kley W., 2016, MNRAS, 459

[16] Meyer D. M.-A., Langer N., Mackey J., Velázquez P. F., Gusdorf A., 2015, MNRAS, 450

[17] Meyer, D. M. -A., Petrov, M. ; Pohl, M. , 2020, MNRAS, 493, 3

[18] Jun B.-I., Norman M. L., 1996, ApJ, 472, 245

[19] Dullemond C. P., Astrophysics Source Code Library, Software 1202.015

[20] Harvey-Smith L., Gaensler B. M., Kothes R., Townsend R., Heald G. H., Ng C. -Y., Green A. J., 2010, ApJ, 712, 2

[21] Kothes R., Foster T. , 2012, ApJ Letters, 746, L4 